\title{
A Radar Architecture for Joint Dynamic Spectrum Access and Target-Matched Illumination
}

\author{
Riccardo Palamà, Hugh Griffiths \\ Electronic and Electrical Engineering Department \\ University College London \\ London, UK \\ \{r.palama,h.griffiths\}@ucl.ac.uk
}

\author{
Francis Watson \\ Defence Science and Technology Laboratory \\ Porton Down, UK
}

fmwatson@dstl.gov.uk

\begin{abstract}
In this study, we address a simple cognitive architecture to design a radar waveform that fills the gaps in the radio-frequency spectrum and matches the target scattering properties. The metric used to calculate the radar waveform is the Mutual Information between the received signal and the target impulse response. Particular attention is paid to analyse the impact of the properties of the communication signals on the chosen metric, for both ideal and non-ideal cases.
\end{abstract}

Keywords-cognitive radar; dynamic spectrum access; targetmatched illumination; waveform design

\section{INTRODUCTION}

Cognitive radar involves a perception-action cycle with memory which is updated as the sensor learns about the target scene [1]. The concept of cognitive radar was first proposed by Haykin [2], with subsequent work by Guerci [3] and others. The need for a cognitive radar system is justified by the requirements of radar users in a modern complex environments, often referred to as Congested-Contested (CC) environment, which includes the presence of communication systems, jamming, other radar users. This environment may change dynamically, and the cognitive radar should be able to respond in real-time to optimise performance, by adapting a wide range of parameters and functions, such as transmit waveform, polarization, carrier frequency, bandwidth, power, scan-rate, dwell time and array configuration.

Recently, portions of the radio-frequency (RF) spectrum previously allocated to radars have been (or will be) allocated to communication systems [4]. In situations where the spectrum occupation by the primary users is not continuous in time, cognitive systems are meant to be used to access the spectrum dynamically, i.e. adapting the transmitted waveform to time-frequency holes. On the other hand, a radar system is designed to detect, track and identify targets, thus the radar waveform should be able not only to avoid the primary user interference, but also to illuminate one or more radar targets in order to optimize the radar performance.

Transmitter adaptivity has been a topic of wide interest in the last years [5], addressing the optimisation of the transmitted waveform based on the maximisation of a chosen metric. Most works in literature have chosen the ratio between the signal and interference-plus-noise power (SINR) as optimisation metric, giving radar waveforms which maximise the detection performance [6].

Previous works that relate to information theory have used the Mutual Information (MI) between the received signal and the target impulse response. The application of information theory to radars was first proposed in the 1950s by Woodward [7], then deepened rather more recently by Bell [8]. The use of MI as a metric is motivated by the fact that it enhances a wide number of spectral components, resulting in a better accuracy in identifying and classifying a radar target. Recent works have addressed the design of radar waveforms that maximise the Mutual Information. In [9] the authors address a waveform optimization technique to adapt to both to signal-dependent and signal-independent disturbance. The MI approach has been considered to address the coexistence between radar and communication systems [10]. In general, the idea of adapting the transmit waveform to the target characteristics is known as "target-matched illumination" [11]

In this work, we propose a processing scheme to realize a joint dynamic spectrum access and target-matched illumination. The proposed scheme is based on the estimation of the PU interference spectrum, followed by an MI-based waveform design block (which exploits the knowledge of the target spectral signature). The dynamic spectrum access is realized by using spectral constraints, which depends on the estimated PU interference spectrum. This design is similar to underlay cognitive-radio architectures [12].

The proposed scheme is validated through simulations, by which we analyse the impact of the primary user interference and of the target properties on the radar performance. The results of simulations of the adopted scheme will be shown and compared with the ideal case, where the interference spectrum is perfectly known. Particular attention will be paid on characterizing the performance of the proposed waveform design technique in situations where the interference spectrum occupancy (SO) increases and its pattern changes. Furthermore, the impact of the observation time (of the fasttime recordings) is studied. This parameter is fundamental to design a real architecture for waveform design, as it is expected that a longer observation time yields a higher accuracy for the estimation of the interference spectrum, thus the resulting waveform gives higher values of our chosen metric. This paper is organized as follows: Sec.II illustrates 
the received signal model, Sec.III addresses the system architecture, whereas the performance analysis is carried out in Sec. IV. Final remarks are drawn in Sec. V.

\section{RECEIVED SIGNAL MODEL}

We assume that the radar is transmitting a waveform $x(t)$, of duration $T_{x}$. The target impulse response is $h_{t}(t)$, of duration $T_{h}$, whereas the interference signal $r_{I}(t)$ is the sum of the signals transmitted by the primary users and received by the radar. Finally, the thermal noise is indicated as $n(t)$. The resulting received signal model is

$$
\begin{aligned}
z(t) & =r(t)+r_{I}(t)+n(t) \\
& =h_{t}(t) \otimes x(t)+r_{I}(t)+n(t)
\end{aligned}
$$

where $\otimes$ is the convolution operator. Hence the Power Spectral Density (PSD) of the received signal is

$$
\begin{aligned}
S_{Z}(f) & =S_{R}(f)+S_{I+N}(f) \\
& =\left|H_{t}(f)\right|^{2}|X(f)|^{2}+S_{I}(f)+S_{N}(f)
\end{aligned}
$$

where $H_{t}(f)$ is the target transfer function.

\section{A. Extended Target Model}

The target signature is due to the different scatterers of a radar target located at different range cells. A radar target that occupies more than one range cell is usually referred to as extended target. Based on the Geometrical Theory of Diffraction (GTD), the model of the target transfer function, used in this work, is given by ([13]) the sum of the contributions from a fixed number of scatterers (indicated as $M$ ), located at different range cells. The total number of scatterers is obtained by dividing the target length along the radial dimension, $L_{t}$, by the radar range resolution, $\Delta R=$ $c / 2 B_{T}$, i.e.

$$
M=2 L_{t} B_{T} / c .
$$

The scatterer index is denoted by $m$ (thus $m=1, \ldots, M$ ). Then the target transfer function is given by

$$
H_{t}(f)=\sum_{m=1}^{M} A_{m}\left(j \frac{f}{f_{c}}\right)^{\alpha_{m}} \exp \left(-j \frac{4 \pi f}{c} r_{m}\right)
$$

where $A_{m}, \alpha_{m}$, and $r_{m}$ are the $m$-th scatterer parameters. The parameter $r_{m}$ indicates the range, and $A_{m}$ is the complex amplitude of the backscattered electric field. The dimensionless parameter $\alpha_{m}$ is related to the geometry of the $m$-th scatterer, assuming value of -1 in the case of corner diffraction, -0.5 for edge diffraction, 0 for a point scatterer, 0.5 for a singly curved surface reflection, 1 for a dihedral-type reflection.

The target PSD is given by $S_{t g t}(f)=\left|H_{t}(f)\right|^{2}$, and the total target power, $P_{t g t}$ is given by integrating the target PSD over all the frequencies. It should be noted that $P_{t g t}$ does not depend on the transmit power, but only on the target scattering phenomena. The value of $P_{t g t}$ could be modified in order to account for the path loss. Fig.1 illustrates an example of extended target PSD, obtained by modelling a target occupying 4 range cells. The total RF bandwidth $\left(B_{T}\right)$ and the sampling frequency $\left(f_{S}\right)$ are both set as $200 \mathrm{MHz}$, thus the radial length of the modelled target is about 3 meters, calculated from eq.(3). The values of the complex amplitude $A_{m}$ are set equal to 1 , whereas the values of the geometrical parameter, $\alpha_{m}$ - collected in the vector $\boldsymbol{\alpha}$ - were chosen randomly, i.e. $\alpha=[1,0,-0.5,0]$. In this study, we assume perfect knowledge of the target PSD. As an alternative, the target PSD could be estimated [14] or extracted from a knowledge database [15].

\section{B. Interference from Communication Systems}

In the hypothesized scenario, the signal transmitted by the primary users and received by the radar is the interference signal, $r_{I}(t)$. We suppose that the interference signal changes with a fixed period, i.e. $\mathrm{T}_{\mathrm{PU}}$, (therefore we consider the processing time required by the proposed technique is smaller than $\mathrm{T}_{\mathrm{PU}}$ ). For a fixed time slot, the number of active primary users is $N$, with $i$ as their index (thus $i=1, \ldots, N$ ). The signal transmitted by the $i$-th PU and received by the radar is

$$
r_{i}(t)=\gamma_{i}(t) \otimes p_{i}(t) \exp \left(j 2 \pi f_{i} t\right)
$$

where $p_{i}(t)$ is the transmitted communication waveform, $\gamma_{i}(t)$ is the channel impulse response, which can be modelled as a Gaussian stochastic process, whose PSD is $S_{\gamma_{i}}(f)$ (in this work the PSD of the communication channel is assumed as constant, modelling the case of flat fading). Finally, $f_{i}$ is the central frequency of the $i$-th PU.

Hence, for the $k$-th time slot, $t \in\left[k T_{P U},(k+1) T_{P U}\right]$, the total primary user interference signal is

$$
r_{I}(t)=\sum_{i=1}^{N} r_{i}(t)=\sum_{i=1}^{N} \gamma_{i}(t) \otimes p(t) \exp \left(-j 2 \pi f_{i} t\right)
$$

whose PSD is

$$
S_{I}(f)=\sum_{i=1}^{N} S_{\gamma_{i}}(f)\left|P\left(f-f_{i}\right)\right|^{2}
$$

The total interference power, $P_{I}$ is given by integrating $S_{I}(f)$ over all the frequencies. A basic choice for the waveform, $p_{i}(t)$, transmitted by each primary user is the sinc one, whose Fourier transform, $P_{i}(f)$, is a rectangular window, of width $B_{i}$.

More realistic choices for $P_{i}(f)$ are the typical spectra of telecommunication systems. Recent communication systems such as 4G and Long Term Evolution (LTE) - are based on Orthogonal Frequency Division Multiplexing (OFDM). However, the interference signal is sampled by the radar receiver at a sampling frequency equal to $f_{S}$, and that the signal is recorded (in fast time) during an interval equal to $T_{o b s}$. Hence the interference spectrum has, in real cases, a bandwidth equal to $B_{i}+1 / T_{o b s}$, due to the spectrum widening due to the finite observation time. Fig. 1 shows the interference spectrum, in the case that it occupies ten channels, centered (in 
base-band) between $-100 \mathrm{MHz}$ and $80 \mathrm{MHz}$, with a step of 20 $\mathrm{MHz}$ and a channel bandwidth of $8 \mathrm{MHz}$ (in the ideal case that the interference spectrum is rectangular-shaped).

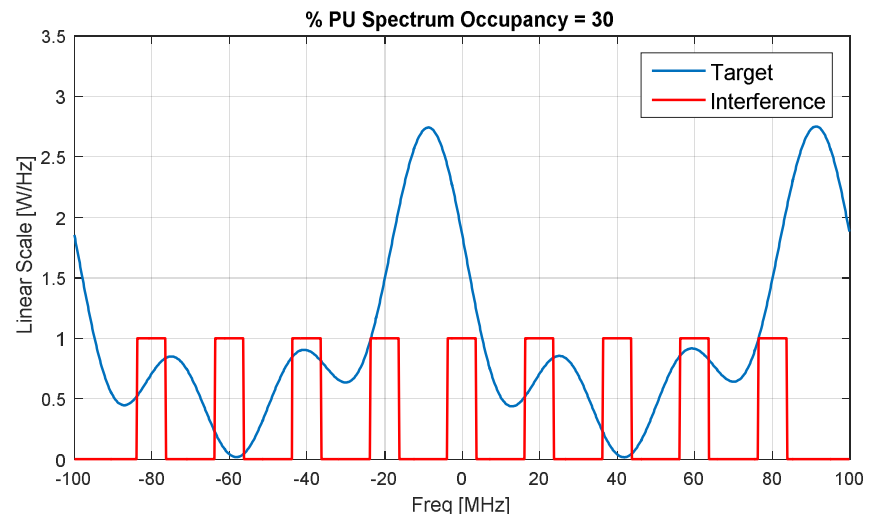

Fig. 1 - Target (blue) and interference (red) spectrum, channel bandwidth $B=$ $8 \mathrm{MHz}$.

\section{PRoposed TeChNiQue}

In this section we illustrate the waveform optimization technique, upon which the general processing scheme described in the second subsection - is based.

\section{A. Waveform Optimization}

In this sub-section we illustrate the waveform optimization method that is the basis of the technique for joint dynamic spectrum access and target-matched illumination proposed in this work. The inputs for the waveform optimization problem are the spectra of the target, interference and noise.

We define the SINR spectral density, $\Psi(f)$, as the ratio between the power spectral densities of the received target signal and of the disturbance, i.e. $\Psi(\mathrm{f})=S_{R}(f) / S_{I+N}(f)$. In the case that both the target signal and disturbance are Gaussian random processes, the Mutual Information between the received signal $z(t)$ and the target impulse response $h_{t}(t)$, given the transmitted waveform $x(t)$ [8], [9], [16], is

$$
\begin{aligned}
I_{M}\left(z(t), h_{t}(t) ; x(t)\right) & =T_{o b s} \int_{B_{T}} \log (1+\Psi(\mathrm{f})) \mathrm{df} \\
& =T_{o b s} \int_{B_{T}} \log \left(1+\frac{\left|H_{t}(f)\right|^{2}|X(f)|^{2}}{S_{I+N}(f)}\right) \mathrm{df}
\end{aligned}
$$

The observation time, $T_{o b s}$ is the temporal interval during which the received signal is recorded (in fast-time), and we assume that the observation time is much greater than both the pulse width $T_{p}$ and the duration of the target impulse response $T_{h}$. Furthermore, since the radio-frequency bandwidth is fixed $\left(B_{T}\right)$, the sampling frequency is also fixed according to the Nyquist criterion for pass-band signals, i.e. $f_{S}=B_{T}$. The number of discrete samples resulting from sampling the received signal by a rate equal to $f_{S}$, is $K=T_{\text {obs }} f_{S}$. The bandwidth $B_{T}$ contains $K$ frequency samples, thus the frequency resolution is equal to $B_{T} / K$. The discrete-time expression of $I_{M}$ is given by

$$
I_{M}(\mathbf{z}, \boldsymbol{h} ; \boldsymbol{x})=\frac{1}{K} \sum_{k=0}^{K-1} \log \left(1+\frac{\left|H_{t}(k)\right|^{2}|X(k)|^{2}}{S_{I}(k)+\sigma^{2}}\right)
$$

The relation between the mutual information and the target classification performance has been first discussed in [8], where the author as derives a simple formula relating the number of target-signal classes that could be "recognized", $N_{C}$, as a function of $\mathrm{I}_{\mathrm{M}}$, i.e.

$$
N_{C}=\left\lfloor\exp \left(I_{M}\right)\right\rfloor
$$

where the operator $\lfloor$.$\rfloor indicates the floor function. It should be$ noted that $N_{C}$ represents the maximum number of equiprobable partitions into which the target signature, $h_{t}(t)$, can be assigned, based on the received signal. The actual target classification performance depends heavily on the mapping of the $N_{C}$ partitions to the set of target classes which are of interest for the radar user. This mapping is related to the particular target classification algorithm employed. In order to give an insight into the potentially achievable classification performance, we use $N_{C}=2$ as the minimum value of the target-signal partitions, thus the minimum value of MI is equal to $\log (2) \cong 0.69$ nats. In this study, we consider the following constraints to the waveform optimization problem:

1) Total transmitted power equal to a desired value, $P_{T}$, thus $\frac{1}{K} \sum_{k=0}^{K-1}|X(k)|^{2}=P_{T}$ Then we define the vector $\boldsymbol{x}_{\boldsymbol{F}}=$ $\left[|X(0)|^{2} \cdots|X(K-1)|^{2}\right], \quad$ hence the constraint becomes $\mathbf{1}^{T} \boldsymbol{x}_{\boldsymbol{F}} \leq K P_{T}$.

2) The power associated with the $k$-th spectral sample must not exceed the maximum value, $c_{k}$, thus $|X(k)|^{2} \leq c_{k} \quad k=$ $0, \ldots, K-1$, summarized by: $\boldsymbol{x}_{F} \preccurlyeq \boldsymbol{c}$, where $\boldsymbol{c}=\left[c_{0} \ldots c_{K-1}\right]$, and the symbol $\preccurlyeq$ indicates the element-wise 'smaller-orequal' operator.

The $c_{k}$ values may be set either automatically or manually. For instance, $c_{k}$ could be set as a function of the output of the spectrum sensing functionality. On the other hand, external factors (of different nature from the spectrum occupancy of the primary user) may influence the setting of the $c_{k}$ constraints, such as the presence of other radar systems working (at least partially) at the same band as the radar under design. In the case that the $k$-th frequency sample has no power limit, its $c_{k}$ value is set as $P_{T}$. The optimization problem consists of finding the optimal waveform $\tilde{x}(t)$, whose squareamplitude spectral values are collected in the vector $\widetilde{\boldsymbol{x}}_{F}$, such that

$$
\begin{aligned}
& \widetilde{\boldsymbol{x}}_{\boldsymbol{F}}=\underset{\boldsymbol{x}_{\boldsymbol{F}}}{\operatorname{argmax}} I(\mathbf{z}, \boldsymbol{h} ; \boldsymbol{x}) \\
& =\underset{\boldsymbol{x}_{\boldsymbol{F}}}{\operatorname{argmax}}\left\{\frac{1}{K} \sum_{k=0}^{K-1} \log \left(1+\frac{\left|H_{t}(k)\right|^{2}|X(k)|^{2}}{S_{I}(k)+\sigma^{2}}\right)\right\} \\
& \text { s.t. } \quad \begin{array}{l}
\mathbf{1}^{T} \boldsymbol{x}_{\boldsymbol{F}}=K P_{T} \\
\quad
\end{array}
\end{aligned}
$$

In the following, we indicate $h_{k}=\left|H_{t}(k)\right|^{2}, x_{k}=x_{F}(k)=$ $|X(k)|^{2}, s_{I, k}=S_{I}(k)$ and $\sigma^{2}$ is the thermal noise power. 


\section{Solution}

The optimization problem in

(11) can be solved by using the Method of Lagrange Multipliers, where the Lagrangian is given by

$$
\begin{aligned}
& L\left(x_{k}\right)=-\frac{1}{K} \sum_{k=0}^{K-1} \log \left(1+\frac{h_{k} x_{k}}{s_{I, k}+\sigma^{2}}\right) \\
& +\delta\left(\sum_{k=0}^{K-1} x_{k}-K P_{T}\right)-\sum_{k=0}^{K-1} \lambda_{k} x_{k}+\sum_{k=0}^{K-1} \gamma_{k}\left(x_{k}-c_{k}\right)
\end{aligned}
$$

We define $b_{k}=\left(s_{I, k}+\sigma^{2}\right) / h_{k}$, representing the spectral density of the interference-to-target ratio $\left(\mathrm{ITR}_{\mathrm{k}}\right)$, then we equate to zero the derivative of $L\left(x_{\mathrm{k}}\right)$ on $x_{\mathrm{k}}$ i.e. $\frac{\partial}{\partial x_{k}} L\left(x_{k}\right)=0$, yielding

$$
\tilde{x}_{k}+b_{k}=\left[K\left(\delta-\lambda_{k}+\gamma_{k}\right)\right]^{-1}
$$

The remaining Karush-Kuhn-Tucker (KKT) conditions are: $\lambda_{k} \tilde{x}_{k}=0, \gamma_{k}\left(\tilde{x}_{k}-c_{k}\right)=0,0 \leq \tilde{x}_{k} \leq c_{k}$. In the case that $0<\tilde{x}_{k}<c_{k}$, then $\lambda_{k}=\gamma_{k}=0$, and $\tilde{x}_{k}=\frac{1}{K \delta}-b_{k}$. Let the constant level be $A=(K \delta)^{-1}$, then the optimal waveform is given by

$$
\tilde{x}_{k}=\left\{\begin{array}{cc}
0 & \text { if } b_{k}>A \\
A-b_{k} & \text { if } A-c_{k} \leq b_{k} \leq A \\
c_{k} & \text { if } b_{k}<A-c_{k}
\end{array}\right.
$$

The value of the constant level, $A$, is found by forcing the constraint on the total transmit power being equal to $P_{T}$ and solving numerically the equation: $\frac{1}{K} \sum_{k=0}^{K-1} \tilde{x}_{k}=P_{T}$. The derived solution is known also as water-filling solution [10]. If there is no limit $c_{k}$ and $b_{k}$ is smaller than the "water level" $A$, the value of $\tilde{x}_{k}$ fills the gap between $b_{k}$ and $A$, which means that the optimal waveform enhances those spectral components for which the energy back-scattered by the target is higher than the interference-plus-noise power (i.e. where $b_{\mathrm{k}}$ is smaller).

\section{B. System Architecture}

Fig.2 illustrates the block diagram of the system considered in this work, addressing the case that the interference signal and the noise level are unknown, and that the target and clutter transfer functions are known. The first block estimates the spectrum of the interference-plus-noise signal, $r_{I}(t)$. The spectrum estimation is realized by recoding the interferenceplus-noise signal for a fixed time interval $\left(T_{o b s}\right)$, during which the radar does not transmit or, in general, interfere with the primary users' signal. The spectrum estimation was realized by using the Welch's method (i.e. the non-parametric averaged periodogram), which is one of the most used, even if basic, spectrum estimates. Many recent works have addressed spectrum estimation techniques, using, for instance the compressive sensing paradigm and the prediction of the dynamics of the spectrum usage by the primary users [17]. The problem of estimating the spectrum of the primary users' signal has been widely addressed in the cognitive radio literature, with the name of 'spectrum sensing'.

After the PSD of the interference-plus-noise signal has been estimated - we indicate this estimate as $\hat{S}_{I+N}(f)$-, the waveform design block computes the optimal waveform (i.e. its PSD, $\left.|X(f)|^{2}\right)$ that maximises the chosen metric. Then the radar signal is transmitted, passes through the radar channel, then it is backscattered (by the radar target) to the radar receiver. The received signal $z(t)$ is then processed to realize one of the several possible radar functions (detection, tracking..). The last block estimates the spectrum of $z(t)$, $\hat{S}_{Z}(f)$, which is necessary to measure the "actual" Mutual Information metric,

$$
\widehat{I_{M}}=\frac{1}{K} \sum_{k=1}^{K} \log \left(\frac{\hat{S}_{Z}(k)}{\hat{S}_{I+N}(k)}\right)
$$

The values of MI calculated from the spectrum estimates $\hat{S}_{I+N}(f)$ (obtained from the first processing stage of Fig.2), and $\hat{S}_{Z}(f)$, are expected to be lower than the ones obtained in the case that the interference signal is known. This is due to the fact that the accuracy of the spectrum estimates $\hat{S}_{I+N}(f)$ and $\hat{S}_{Z}(f)$ depends on the number of samples, $K$., and on the interference and target characteristics. The impact of the observations time, and of the number of samples, $\mathrm{K}$, will be analysed in the next sections.

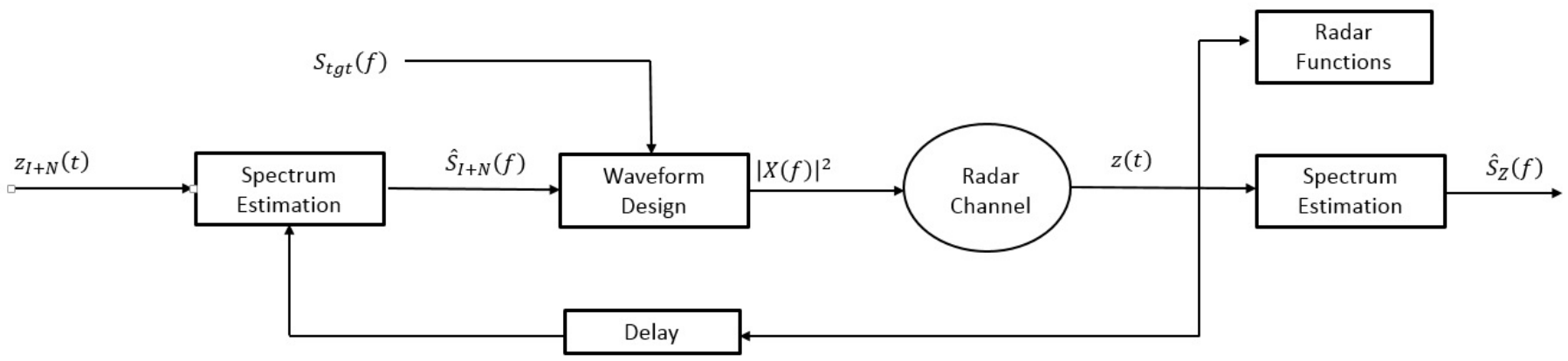

Fig. 2 - Block diagram of the system architecture 


\section{PERFormance ANALYSIS}

In this section, we report the results obtained by simulating the scheme illustrated in Fig.2. The interference signal was generated as a coloured Gaussian random process, whose spectrum was synthesized according to the interference model. We considered the case that the available bandwidth is $200 \mathrm{MHz}$, the interference is centred on [-80 -20 0 60] MHz. The channel bandwidth $\left(B_{i}\right)$ was gradually increased from $400 \mathrm{kHz}$ to $15 \mathrm{MHz}$, which yields a spectrum occupancy between $2 \%$ and $30 \%$. The thermal-noise power was fixed, equal to $1 \mathrm{~W}$.

The interference spectrum was estimated by employing the Welch's estimation method with a moving window of 64 temporal samples. The target signal was also generated as a coloured Gaussian random process, whose PSD, $S_{t g t}(f)$ (see Fig.1), was assumed as known. The ratio between the target power, $P_{t g t}$, and the interference-plus-noise power $\left(P_{I}+P_{N}\right)$ was set as $0 \mathrm{~dB}$. A series of 100 Monte Carlo runs was realized in order to evaluate the impact of some interference and system parameters, i.e. the observation time, the Interference-to-Noise Ratio $\left(I N R=P_{I} / P_{N}\right)$ and the interference spectrum occupancy (i.e. the percentage of the spectrum occupied by the interference over the total available bandwidth). For each Monte Carlo run, the scheme in Fig. 2 was simulated and the final value of the Mutual Information was calculated, then the MI values were averaged.

\section{A. Observation time}

The parameter $K$ is the total number of "fast-time" samples collected by the radar, and influences heavily the accuracy of the spectrum estimate. Large values of $K$ guarantee a good accuracy of the spectrum estimate, then are expected to give high values of the Mutual Information $\left(\hat{I}_{M}\right)$. Fig. 3 shows a comparison among the waveforms in the simulated case, obtained by using 1024 and 4096 samples, and the theoretical one (if the interference spectrum is rectangular and known). It could be noted that, for a larger number of samples, the resulting waveform is closer to the optimal one, obtained in the ideal case. However, the uncertainty due to a finite observation time and to the random behaviour of the interference signal causes fluctuations of the MI values. The variance of the MI values is analysed for different values of the interference spectrum occupancy, as a function of the number of samples, $K$. Fig.4 shows a decreasing behaviour of the MI variance with increasing number of samples. If the number of samples is smaller than 2048, the variance of the MI values is high. Higher values of the variance of the MI values cause a higher uncertainty in the classification of two or more different target classes.

\section{B. Interference-to-Noise Ratio}

The influence of the interference power is analysed in this section. Fig.5 shows the MI values as a function of the interference-to-noise ratio (INR), for a fixed $\mathrm{K}=4096$. It could be noted that, in the ideal case, the MI values are constant, about 0.6 and 1.67 for $\mathrm{P}_{\mathrm{T}}$ equal to $1 \mathrm{~W}$ and $10 \mathrm{~W}$, respectively. In the simulated case, the MI values decrease with increasing INR, with the slope of this decreasing curve influenced by the transmit power. This decreasing behaviour is possibly due to the side-lobes of the interference spectrum that appear in realistic cases. The level of the side-lobes increases with increasing interference power and tends to "saturate" the white spaces in the RF spectrum that the radar waveform would fill. Furthermore, the loss in the MI value is also more evident in the case that the transmit power is high. In other words, the gain given by increasing the transmit power seems to decrease if the interference power increases.

\section{Comparison with non-optimal waveform}

The proposed method assumes the perfect knowledge of the target signature and, once estimated the interference spectrum, designs the waveform that maximizes the Mutual Information. In order to compare the performance of the proposed method with a basic one, the waveform is computed in the case that the target signature is supposed, erroneously, as flat (the obtained waveform will be referred to as "non-optimal"). The resulting non-optimal waveform notches the sub-bands where the interference is present thus the "coexistence" between radar and communication systems is preserved-, while giving equal weight to the remaining sub-bands. Fig.6 shows the comparison between the MI values obtained by using the optimal and the nonoptimal waveforms, as a function of the interference spectrum occupancy (SO). An interference model with ten occupied channels was employed in these simulations, yielding a maximum PU spectrum occupancy of about $70 \%$. In general, the MI values obtained by using the optimal waveform are higher than the ones obtained by using the non-optimal waveform. In the theoretical case (where the interference spectrum is rectangular and known), a MI equal to 0.85 nats is obtained for a SO of about $42 \%$ and $35 \%$, for the optimal and non-optimal waveform, respectively. In the simulated case (using 4096 samples), an average MI of about 0.85 nats is obtained for a SO of about $27 \%$ and $10 \%$, for the optimal and non-optimal waveform, respectively. However, it should be noted that, with an increasing spectrum occupancy, the gap between the performance of the optimal and non-optimal waveforms decreases, and for a spectrum occupancy greater than $65 \%$, using the optimal waveform does not provide any gain. This is probably due to the fact that the proposed optimization is a constrained one, meaning that the computed solution is not (in some particular situations) the global optimum.

\section{CONCLUSION}

In this study, we have addressed a simple cognitive architecture to design a radar waveform that fills the gaps in the RF spectrum and matches the target scattering properties. The proposed method has shown quite good 
performance, giving values of the MI higher than 0.7 (which is the minimum theoretical value in order to recognize two target classes), for values of the PU spectrum occupancy up to $60 \%-65 \%$, if the transmit power is properly chosen.

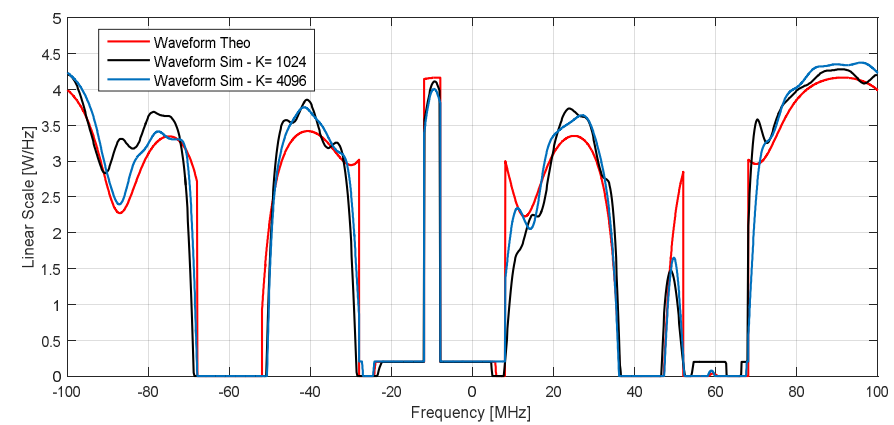

Fig. 3 - Example of designed waveform in the theoretical case (red), and simulated, with variable number of samples (K).

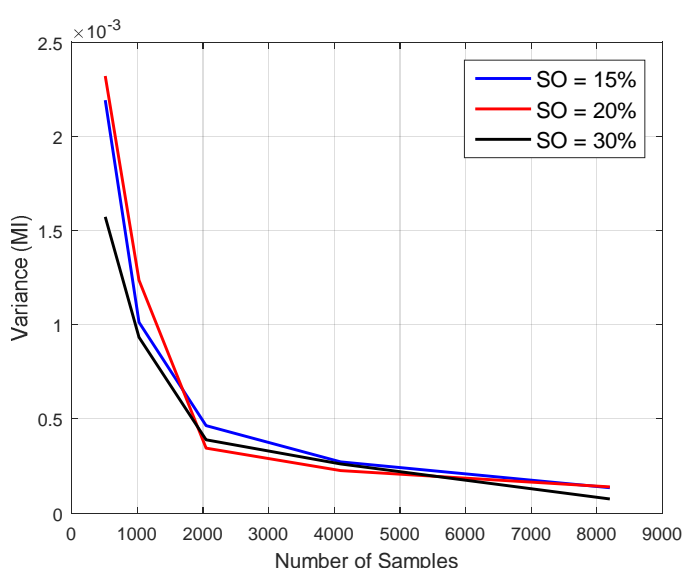

Fig. 4 - Variance of the MI values in the simulated case, as a function of the number of samples, for different values of the spectrum occupancy (SO)

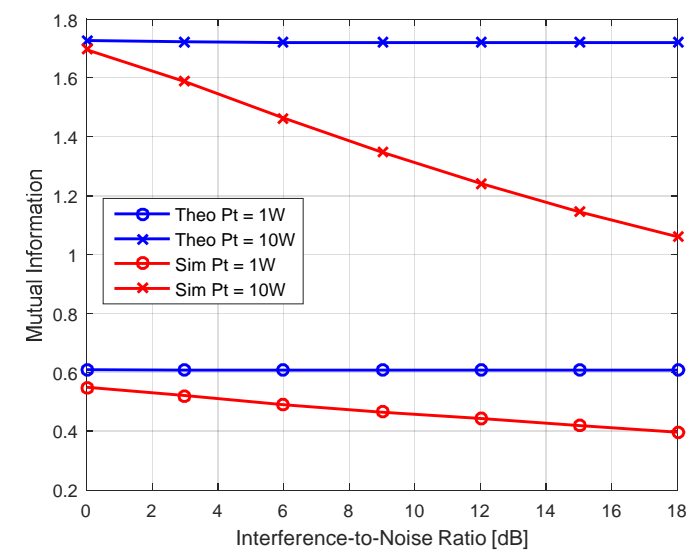

Fig. 5 - Mutual Information (nats) as a function of the INR (dB) spectrum occupancy of 30\%, for different values of the transmit power. Comparison between the values of MI in the theoretical case (known target spectrum) and in the simulated case (estimated target spectrum).

\section{ACKNOWLEDGMENT}

The authors would like to thank DSTL that has made substantial contribution to post-doctoral funds.

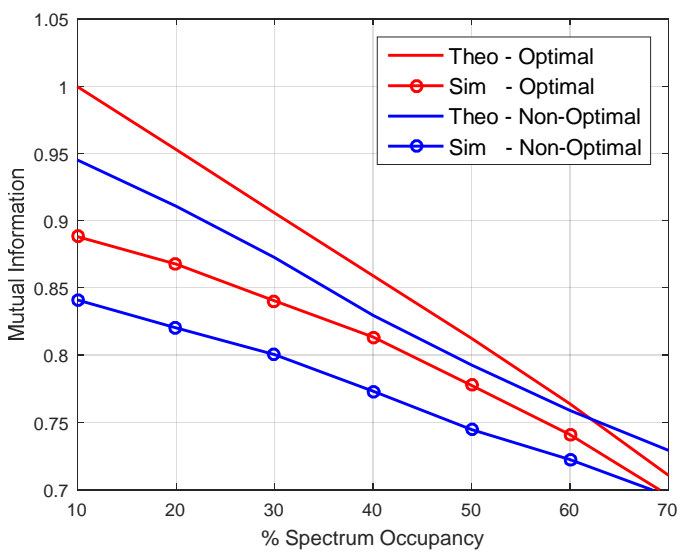

Fig. 6 -- Comparison between the MI values (nats) obtained by using the optimal waveform and the non-optimal one (obtained by assuming a flat target spectrum) as a function of SO, in the theoretical (known target signature) and simulated (estimated target spectrum) cases.

\section{REFERENCES}

[1] S. Haykin, Cognitive Dynamic Systems: Perception-Action Cycle, Radar and Radio. Cambridge University Press, 2012.

[2] S. Haykin, "Cognitive radar: a way of the future," Signal Processing Magazine, IEEE, vol. 23, no. 1. pp. 30-40, 2006.

[3] J. R. Guerci, Cognitive Radar: The Knowledge-Aided Fully Adaptive Approach. Artech House, 2010.

[4] H. Griffiths, L. Cohen, S. Watts, E. Mokole, C. Baker, M. Wicks, and S. Blunt, "Radar Spectrum Engineering andManagement: Technical and Regulatory Issues," Proc. IEEE, vol. 103, no. 1, pp. 85-102, 2015.

[5] F. Gini, A. De Maio, and L. Patton, Waveform design and diversity for advanced radar systems. London: IET, 2012.

[6] A. Aubry, A. De Maio, A. Farina, and M. Wicks, "KnowledgeAided (Potentially Cognitive) Transmit Signal and Receive Filter Design in Signal-Dependent Clutter," IEEE Trans. Aerosp. Electron. Syst., vol. 49, no. 1, pp. 93-117, 2013.

[7] P. M. Woodward, Probability and Information Theory with application to radar. 1953.

[8] M. R. Bell, "Information Theory and Radar Waveform Design," IEEE Trans. Inf. Theory, vol. 39, no. 5, pp. 1578-1597, 1993.

[9] R. a. Romero and N. a. Goodman, "Waveform design in signaldependent interference and application to target recognition with multiple transmissions," IET Radar, Sonar Navig., vol. 3, no. 4, p. 328,2009 .

[10] M. Bica and V. Koivunen, "Generalized Multicarrier Radar: Models and Performance," IEEE Trans. Signal Process., vol. 64 no. 17, pp. 4389-4402, 2016.

[11] A. Farina and F. A. Studer, "Detection with high resolution radar: great promise, big challenge," Microw. J., pp. 263-273, 1991.

[12] A. Goldsmith, S. A. Jafar, I. Maric, and S. Srinivasa, "Breaking Spectrum Gridlock With Cognitive Radios: An Information Theoretic Perspective," Proc. IEEE, vol. 97, no. 5, pp. 894-914, 2009.

[13] L. C. Potter, D. M. Chiang, R. Carriere, and M. J. Gerry, "A GTD-Based Parametric Model for Radar Scattering," IEEE Trans. Antennas Propag., vol. 43, no. 10, pp. 1058-1067, 1995.

[14] Y. Chen, Y. Nijsure, and C. Yuen, "Adaptive Distributed MIMO Radar Waveform Optimization Based on Mutual Information," IEEE Trans. Aerosp. Electron. Syst., vol. 49, no. 2, pp. 1374$1385,2013$.

[15] F. Gini and M. Rangaswamy, Knowledge Based Radar Detection, Tracking and Classification. Wiley, 2008.

[16] R. A. Romero, J. Bae, and N. A. Goodman, "Theory and application of SNR and mutual information matched illumination waveforms," IEEE Trans. Aerosp. Electron. Syst., vol. 47, no. 2, pp. 912-927, 2011.

[17] P. Stinco, M. S. Greco, and F. Gini, "Spectrum sensing and sharing for cognitive radars," IET Radar, Sonar Navig., vol. 10, no. 3, pp. 595-602, 2016. 